\title{
Structural MRI research in patients with nasopharyngeal carcinoma following radiotherapy: A DTI and VBM study
}

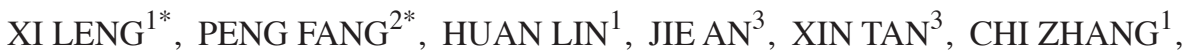 \\ DONGLIN WU ${ }^{3}$, WEN SHEN ${ }^{1}$ and SHIJUN QIU ${ }^{1}$

\begin{abstract}
${ }^{1}$ Medical Imaging Center, Nanfang Hospital, Southern Medical University, Guangzhou, Guangdong 510515; Guangzhou, Guangdong 510405, P.R. China
\end{abstract} \\ ${ }^{2}$ College of Mechatronics and Automation, National University of Defense Technology, Changsha, Hunan 410073; \\ ${ }^{3}$ Medical Imaging Center, The First Affiliated Hospital of Guangzhou University of Traditional Chinese Medicine,
}

Received June 10, 2016; Accepted April 26, 2017

DOI: $10.3892 / \mathrm{ol} .2017 .6968$

\begin{abstract}
The aim of the present study was to investigate the microstructural characteristics of the brain lobes following radiotherapy (RT) for patients with nasopharyngeal carcinoma (NPC) at distinct times. Diffusion tensor imaging (DTI) and 3D-T1-weighted imaging was performed in 70 age- and sex-matched subjects, 24 of whom were pre-treatment patients. The patients were divided into three groups, according to the time following completion of RT. Fractional anisotropy (FA) and gray matter (GM) volume were determined. The DTI data were analyzed using tract-based spatial statistics and the GM volume was analyzed using voxel-based morphometry (VBM). Compared with the pre-RT group, the mean FA values in the left parietal lobe white matter (WM) and right cerebellum decreased significantly in the post-RT 0-6 month group $(\mathrm{P}<0.05)$. In addition, the mean FA values in the right parietal lobe WM decreased significantly in the post-RT 6-12 month group $(\mathrm{P}<0.05)$, compared with the pre-RT group. The FA level in the right temporal lobe remained significantly decreased, compared with that in the pre-RT group $(\mathrm{P}<0.05)$ for 1 year after RT. Furthermore, compared with pre-RT group, the GM volume in the bilateral frontal lobe, right occipital lobe, left parietal lobe, right temporal lobe and left cerebellum decreased significantly in the post-RT 0-6 month group $(\mathrm{P}<0.05)$, and in the bilateral temporal lobe, parietal
\end{abstract}

Correspondence to: Professor Shijun Qiu, Medical Imaging Center, Nanfang Hospital, Southern Medical University, 1838 North Avenue, Guangzhou, Guangdong 510515, P.R. China

E-mail: qiushijun666@163.com

${ }^{*}$ Contributed equally

Abbreviations: RT, radiotherapy; NPC, nasopharyngeal carcinoma; DTI, diffusion tensor imaging; TBSS, tract-based spatial statistic; WM, white matter; VBM, voxel-based morphometry; GM, gray matter; FA, fractional anisotropy

Key words: nasopharyngeal carcinoma, diffusion tensor imaging, tract-based spatial statistic, voxel-based morphometry, fractional anisotropy lobe, right frontal lobe and left cerebellum, the GM volume decreased significantly in the post-RT 6-12 month group $(\mathrm{P}<0.05)$. The GM volume in the right temporal lobe, bilateral frontal lobe and bilateral cerebellum remained significantly decreased compared with that in the pre-RT group $(\mathrm{P}<0.05)$ for 1 year after RT. A combination of DTI and VBM may be used to determine radiation-induced brain injury in patients treated for NPC.

\section{Introduction}

Nasopharyngeal carcinoma (NPC) is a common type of malignant tumor in southern China, with the highest incidence in Guangdong Province (1). Radiotherapy (RT) is the standard treatment method; however, following RT, radiation fibrosis or radiation encephalopathy may occur $(2,3)$. The pathophysiology of radiation encephalopathy is divided into three periods: The acute reaction period, the early delayed radiation period and the late delayed radiation period. Radiation-induced brain injury is irreversible, therefore detection of this in the early stage is important. Traditionally, the white matter (WM) and vascular endothelium have been considered to be key structures for RT-induced brain damage (4). Previous studies have focused on alterations in the WM of the temporal lobe, including metabolic changes and abnormalities of water diffusion in normal-appearing WM in the early phases, using conventional magnetic resonance imaging (MRI) of patients with NPC (5-7).

Magnetic resonance diffusion tensor imaging (DTI) is currently the only non-invasive method used to assess the structure, morphology and function of WM fibers in vivo $(8,9)$. DTI technology has been widely used in research of the central nervous system. Tract-based spatial statistics (TBSS) is a novel processing method of DTI data, with marked advantages compared with the manually drawn regions of interest (ROI) method (10). Previous studies have primarily used the ROI method and have been confined to the temporal lobe (6-7). To the best of our knowledge, the present study was the first to examine the dynamic alterations in the microstructure of whole brain WM in distinct stages of NPC prior to and following RT, using the TBSS method. 
Although WM is more vulnerable to radiation-induced injury, compared with gray matter (GM), previous studies have identified that the GM may be damaged $(11,12)$. Voxel-based morphometry (VBM) is a suitable method used to assess GM volume alterations in vivo. A previous study evaluated radiation-induced GM volume alterations in patients with NPC (13). The present study aimed at determining the microstructural alterations in normal-appearing WM and GM structures in patients with NPC following RT, using conventional MRI.

\section{Patients and methods}

Patients. The present study included 70 subjects (53 males, 17 females; aged between 25 and 59 years; mean age, 44 years) with pathologically confirmed NPC. Of the 70 subjects, 24 were pre-RT and the remaining 46 were post-RT. The time between RT and imaging ranged between 1 week and 4 years. All patients underwent fractionated RT for the first time with three-dimensional conformal and intensity-modulated techniques (total dose/fraction dose/exposures, 66-74 Gy/1.8-2.0 Gy/30-35 times). Prior to MRI examinations, it was validated that patients exhibited no occurrences of intracranial tumors or intracranial invasion. Patients with high blood pressure, diabetes, heart disease, WM degeneration or vascular lesions were excluded.

Pre-RT subjects constituted the control group. Post-RT patients were divided into three groups according to the stage of radiation-induced brain injury which include acute reaction period, early delayed radiation period and late delayed radiation period (7): Group 1 (pre-RT, n=24); group 2 (0-6 months post-RT, $\mathrm{n}=16$ ); group 3 ( $>6-12$ months post-RT, $\mathrm{n}=16$ ); and group 4 ( $>12$ months post-RT, $n=14$ ). No statistically significant differences were identified among the groups according to age or sex. The present study was approved by the Institutional Review Board of Southern Medical University, Nanfang Hospital (Guangzhou, China) and was conducted under strict adherence to the Privacy Rules of The Health Insurance Portability and Accountability Act. All individuals included were fully informed of the purpose, methods and precautions of the trial, and written informed consent was obtained from all participants.

Image acquisition. MRI data were acquired using a $3.0 \mathrm{~T}$ clinical scanner with an eight-channel head coil (SIGNA EXCITE; GE Healthcare, Chicago, IL, USA). The routine MRI brain protocol included axial T1-weighted images [repetition time (TR), $600 \mathrm{~ms}$; echo time (TE), $15 \mathrm{~ms}$, T2-weighted images (TR, 5,200 ms; TE, $140 \mathrm{~ms}$ ) and T2-weighted fluid attenuated inversion recovery (TR, 9,000 ms; TE, $120 \mathrm{~ms}$; inversion recovery, 2,100 ms). DTI scans were performed, employing a single-shot echo-planar imaging sequence and an array spatial sensitivity encoding technique with the following parameters: TR, 12,000 ms; TE, $75.5 \mathrm{~ms}$; field of view (FOV), 24x24 cm; matrix, 128x128; slice thickness, $3 \mathrm{~mm}$ (no inter-slice gap); number of excitation, 1; and flip angle, $90^{\circ}$. Images were collected along 25 non-collinear diffusion gradient directions, with a b-value of $1,000 \mathrm{sec} / \mathrm{mm}^{2}$ and one set of null images with $a b$ value of $0 \mathrm{sec} / \mathrm{mm}^{2}$. 3D-T1 imaging was performed using a three-dimensional fast field echo pulse sequence with the following imaging parameters: TR, 2,500 ms; TE, $1.5 \mathrm{~ms}$; FOV, 24x24 cm; matrix, 256x256; slice thickness, $1 \mathrm{~mm}$; slice gap, $0 \mathrm{~mm}$; NEX, 1.

Post-processing. DTI data were analyzed using the Functional MRI of the Brain (FMRIB) Software Library (FSL) tools (version 4.19; www.firirib.ox.ac.uk/fsl). The protocol used was as follows: i) DTI data were adjusted for head movement and eddy current distortion, using the Diffusion Toolbox of the FSL software; ii) mask images of each brain were obtained using each subject's B0 image with the Brain Extraction Tool; iii) FMRIB Software Library's Diffusion Toolbox was used to calculate the diffusion tensor and fractional anisotropy (FA) maps were obtained; and iv) TBSS processes were monitored (14).

An extension tool of Statistical Parametric Mapping (SPM), the diffeomorphic anatomical registration through exponentiated lie algebra tool, was used to process the GM data. The following procedures were used: i) The original image was segmented to gray matter, white matter and cerebral spinal fluid images and subsequently imported into DARTEL for calculation and obtaining GM images; ii) DARTEL was used to create a GM template; iii) the GM template was used to normalize each subject's GM image; iv) the resulting GM images were modulated with a Jacobian correction; v) a $12 \mathrm{~mm}$ full width at half maximum isotropic Gaussian kernel was used to smooth the resulting images; and vi) the GM data were transformed to Montreal Neurological Institute coordinates for SPM analysis. Subsequently, the preprocessed GM data were analyzed using Statistical parametrical mapping 8 (Wellcome Department of Cognitive Neurology, London, UK, www.fil.ion.ucl.ac.uk/spm), with a general linear model and random field theory.

Statistical analysis. Statistical analysis was performed using SPSS software (version 13.0; SPSS, Inc., Chicago, IL, USA). One-way analysis of variance with multiple comparisons using the Bonferroni post hoc test was used to analyze the differences between age among the groups. Pearson's $\chi^{2}$ test was used to determine sex differences. Threshold-free cluster enhancement in randomize mode (15) was used to analyze FA, with permutation-based correction for multiple comparisons at $\mathrm{P}<0.05$. The differences between GM volumes among the groups were determined using a voxel-based comparison. A cluster-level threshold of $\mathrm{P}<0.05$ were set to produce statistical maps, which were corrected for multiple comparisons using the false discovery rate (FDR).

\section{Results}

$F A$. Compared with the pre-RT group, the mean FA values in the left parietal lobe WM and right cerebellum decreased significantly in the post-RT $0-6$ month group $(\mathrm{P}<0.05$; Fig. 1; Table I), and the mean FA values in the right parietal lobe WM decreased significantly in the post-RT 6-12 month group $(\mathrm{P}<0.05$; Fig. 2; Table II). In the $>12$ months post-RT group, the FA level in the right temporal lobe remained significantly lower for 1 year after RT, compared with that in the pre-RT group ( $\mathrm{P}<0.05$; Fig. 3; Table III). 
Table I. Brain regions with fractional anisotropy values in the 0-6 month post-radiotherapy group of patients with nasopharyngeal carcinoma, compared with those in the pre-radiotherapy group.

\begin{tabular}{lccccc}
\hline & & \multicolumn{4}{c}{$\begin{array}{c}\text { MNI coordinate } \\
(\mathrm{mm})\end{array}$} \\
\cline { 3 - 5 } Brain regions & Voxels & $\mathrm{X}$ & $\mathrm{Y}$ & $\mathrm{Z}$ & P-value \\
\hline Left parietal lobe & 30 & -7 & -57 & -30 & $<0.0001$ \\
Right cerebellum & 24 & 44 & -39 & 18 & $<0.0001$ \\
\hline
\end{tabular}

$\mathrm{P}<0.05$, FWE, family-wise error corrected.

Table II. Brain regions with fractional anisotropy values in the 6-12 month post-radiotherapy group in patients with nasopharyngeal carcinoma, compared with those in the pre-radiotherapy group.

\begin{tabular}{lccccc}
\hline & & \multicolumn{4}{c}{$\begin{array}{c}\text { MNI coordinate } \\
(\mathrm{mm})\end{array}$} \\
\cline { 3 - 5 } Brain region & Voxels & $\mathrm{X}$ & $\mathrm{Y}$ & $\mathrm{Z}$ & P-value \\
\hline Right parietal lobe & 28 & -10 & -28 & 33 & 0.019 \\
\hline
\end{tabular}

$\mathrm{P}<0.05$, FWE, family-wise error corrected.

Table III. Brain regions with fractional anisotropy values in the $>12$ month post-radiotherapy group of patients with nasopharyngeal carcinoma, compared with those in the pre-radiotherapy group.

\begin{tabular}{lccccc}
\hline & & \multicolumn{4}{c}{$\begin{array}{c}\text { MNI coordinate } \\
(\mathrm{mm})\end{array}$} \\
\cline { 3 - 5 } Brain region & Voxels & $\mathrm{X}$ & $\mathrm{Y}$ & $\mathrm{Z}$ & P-value \\
\hline Right temporal lobe & 22 & -43 & -25 & -15 & $<0.0001$ \\
\hline
\end{tabular}

$\mathrm{P}<0.05$, FWE, family-wise error corrected.

GM volume. Compared with the pre-RT group, the GM volume in the bilateral frontal lobe, right occipital lobe, left parietal lobe, right temporal lobe and left cerebellum decreased significantly in the post-RT 0-6 month group $(\mathrm{P}<0.05$; FDR corrected; clusters, $100 \mathrm{~mm}^{3}$; Fig. 4; Table IV). In addition, in the bilateral temporal lobe, parietal lobe, right frontal lobe and left cerebellum, the GM volume decreased significantly in the post-RT 6-12 month group $(\mathrm{P}<0.05$; Fig. 5; Table V), compared with that of the pre-RT group. In the $>12$ month post-RT group, for 1 year after RT, the GM volume in the right temporal lobe, bilateral frontal lobe and bilateral cerebellum remained significantly decreased, compared with that in the pre-RT group $(\mathrm{P}<0.05$; Fig. 6; Table VI).

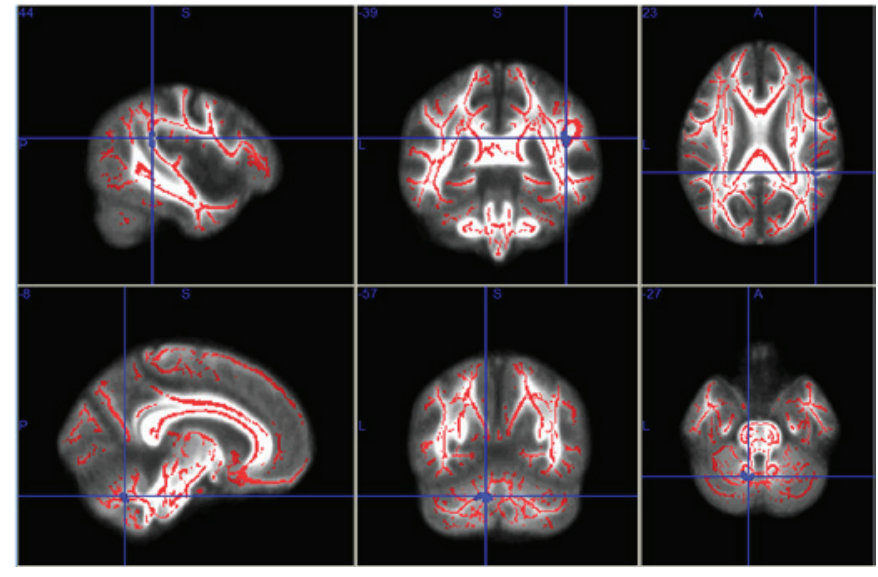

Figure 1. Compared with the pre-radiotherapy group, the fractional anisotropy values in the left parietal lobe white matter and right cerebellum markedly decreased in the 0-6 month post-radiotherapy group.
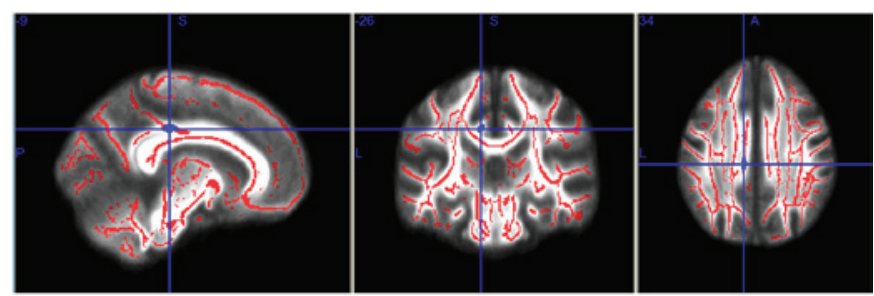

Figure 2. Compared with the pre-radiotherapy group, the fractional anisotropy values in the right parietal lobe white matter markedly decreased in the 6-12 month post-radiotherapy group.

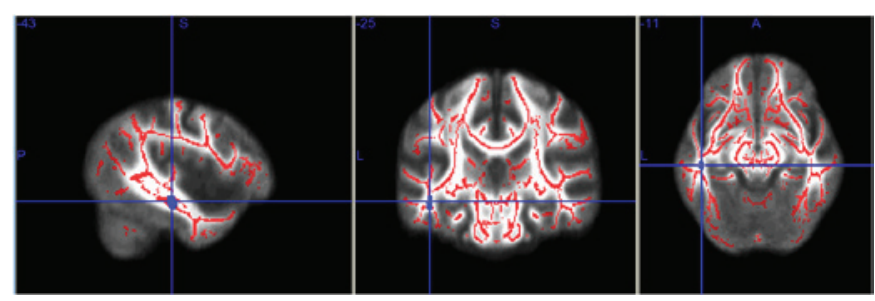

Figure 3. Compared with the pre-radiotherapy group, the fractional anisotropy values in the right temporal lobe white matter markedly decreased in the $>12$ month post-radiation group.

\section{Discussion}

To the best of our knowledge, the present study was the first to analyze the microstructural dynamic alterations in all brain lobes following RT in patients with NPC at different times, using DTI-TBSS for WM and VBM for GM volume. The overall brain alterations were extensive and dynamic, and were not limited to the temporal lobe, which has not been reported previously.

RT is the preferred and most effective treatment for NPC; however, the field of RT contains the temporal lobes and the radiation dose exceeds the brain tissue tolerance. Radiation-induced injury of brain tissue is one of the most serious complications, which may influence the prognosis and decrease the quality of life of patients with NPC. A previous study identified that the incidence of radiation-induced brain 
Table IV. Brain regions with significantly reduced gray matter volume in the 0-6 month post-radiotherapy group of patients with nasopharyngeal carcinoma compared with the pre-radiation group.

\begin{tabular}{lrrrrr}
\hline & & \multicolumn{4}{c}{$\begin{array}{c}\text { MNI coordinate } \\
(\mathrm{mm})\end{array}$} \\
\cline { 3 - 5 } & Voxels & $\mathrm{X}$ & $\mathrm{Y}$ & $\mathrm{Z}$ & P-value \\
\cline { 3 - 5 } Brain region & 102 & 28 & -76 & -56 & $<0.0001$ \\
\hline Right occipital lobe & 110 & -8 & -68 & -38 & $<0.0001$ \\
Left occipital lobe & 134 & -60 & -14 & -36 & $<0.0001$ \\
Right temporal lobe & 117 & -18 & -50 & -2 & $<0.0001$ \\
Left occipital lobe & 187 & 40 & 8 & 22 & $<0.0001$ \\
Right frontal lobe & 187 & & \\
Right temporal lobe & 403 & 6 & 6 & 38 & $<0.0001$ \\
\hline
\end{tabular}

$\mathrm{P}<0.05$, false discovery rate corrected.

Table V. Brain regions with significantly reduced gray matter volume in the 6-12 month post-radiotherapy group of patients with nasopharyngeal carcinoma compared with the pre-radiation group.

\begin{tabular}{lcrrrr}
\hline & \multicolumn{5}{c}{$\begin{array}{c}\text { MNI coordinate } \\
(\mathrm{mm})\end{array}$} \\
\cline { 3 - 5 } & & \multicolumn{3}{c}{ B } & \\
\cline { 3 - 5 } Brain region & Voxels & $\mathrm{X}$ & $\mathrm{Y}$ & $\mathrm{Z}$ & P-value \\
\hline Frontal lobe & 230 & 28 & -74 & -26 & $<0.0001$ \\
Occipital lobe & 110 & -18 & 10 & -26 & $<0.0001$ \\
Temporal lobe & 139 & 40 & -20 & -6 & $<0.0001$ \\
Temporal lobe & 455 & -56 & 6 & -6 & $<0.0001$ \\
Temporal lobe & 222 & 42 & 6 & 4 & $<0.0001$ \\
Frontal lobe & 114 & -4 & 54 & 6 & $<0.0001$ \\
Temporal lobe & 141 & -54 & -32 & 14 & $<0.0001$ \\
Parietal lobe & 218 & 60 & -8 & 18 & $<0.0001$ \\
Frontal lobe & 743 & -54 & -4 & 20 & $<0.0001$ \\
Parietal lobe & 176 & -48 & -58 & 34 & 0.001 \\
Temporal lobe & 199 & -10 & -28 & 40 & $<0.0001$ \\
& & & & &
\end{tabular}

$\mathrm{P}<0.05$, false discovery rate corrected.

injury was between 4 and 18\% (16). Chong et al (17) reported that radiation-induced encephalopathy exhibited a long incubation period. Thus, monitoring the structural alterations in the brain tissue is required.

Alterations in the water diffusion parameters in normal-appearing WM on conventional MRI scans may be assessed quantitatively using DTI. DTI has been widely used in studies of the central nervous system and it has been used to investigate WM injury following cranial irradiation $(18,19)$. FA imaging is most widely used in clinical research due to FA exhibiting the highest Signal to Noise Ratio and the best detail resolution. To the best of our knowledge, the present study was the first time that TBSS was used to process the
Table VI. Brain regions with significantly reduced gray matter volume in the $>12$ month post-radiotherapy group of patients with nasopharyngeal carcinoma compared with the pre-radiation group.

\begin{tabular}{|c|c|c|c|c|c|}
\hline \multirow[b]{2}{*}{ Brain region } & \multirow[b]{2}{*}{ Voxels } & \multicolumn{3}{|c|}{$\begin{array}{l}\text { MNI coordinate } \\
\qquad(\mathrm{mm})\end{array}$} & \multirow[b]{2}{*}{$\mathrm{P}$-value } \\
\hline & & $X$ & $\mathrm{Y}$ & $\mathrm{Z}$ & \\
\hline Left cerebellum & 225 & -42 & -78 & -38 & $<0.0001$ \\
\hline Right cerebellum & 127 & 30 & -70 & -28 & $<0.0001$ \\
\hline Right temporal lobe & 282 & 8 & 10 & -26 & $<0.0001$ \\
\hline Left temporal lobe & 140 & -44 & 6 & -8 & $<0.0001$ \\
\hline
\end{tabular}

$\mathrm{P}<0.05$, false discovery rate corrected.

DTI data of patients with NPC. In addition, previous studies of radiation-induced brain injury have been confined to the temporal lobes, but, as radiation may damage WM in other brain regions, the present study investigated the whole brain.

Previous studies have demonstrated that FA values decreased markedly in the acute phase and early delayed reaction period following $\mathrm{RT}$, and subsequently gradually recovered (6,7). Kitahara et al (19) reported that, following RT in patients with brain tumors, FA values decreased markedly between 3 and 5 months, and subsequently recovered after 6 months. The results of the present study identified that FA values in the left parietal lobe and in the right cerebellar hemisphere WM, of patients between 0 and 6 months after RT, were statistically significantly decreased compared with that of the control group, and this is consistent with previous studies. The aforementioned two brain regions are located close to the temporal lobe and may have been included in the radiation field, resulting in WM damage. In the acute phase and early delayed reaction period, the diffusion rate of water molecules along the nerve fibers decreased due to encephaledema, the demyelination of nerve fibers and the destruction of myelin (19), and the decreased FA values may reflect these pathological alterations in the WM. In the present study, the FA values in the late delayed reaction period remained markedly decreased, compared with that in the pre-RT group. Additionally, the FA values in the right parietal lobe WM markedly decreased in the post-RT 6-12 month group and the values in the right temporal lobe WM markedly decreased after 12 months, compared with that in the pre-RT group. In the late delayed reaction period, as the brain edema was absorbed, the degree of demyelination decreased, myelin regeneration occurred and the FA values in the partial lobe WM recovered gradually (7). In the present study, the FA values in the left parietal lobe and right cerebellar hemisphere WM did not markedly decrease after 6 months and the FA values in right parietal lobe WM did not decrease significantly after 12 months. The results of the present study indicated that the FA values in a number of brain regions increased gradually, which was consistent with previous studies. At the cessation of RT, the pathological alterations in the WM, due to radiation injury, recovered partially. The FA value in the right 


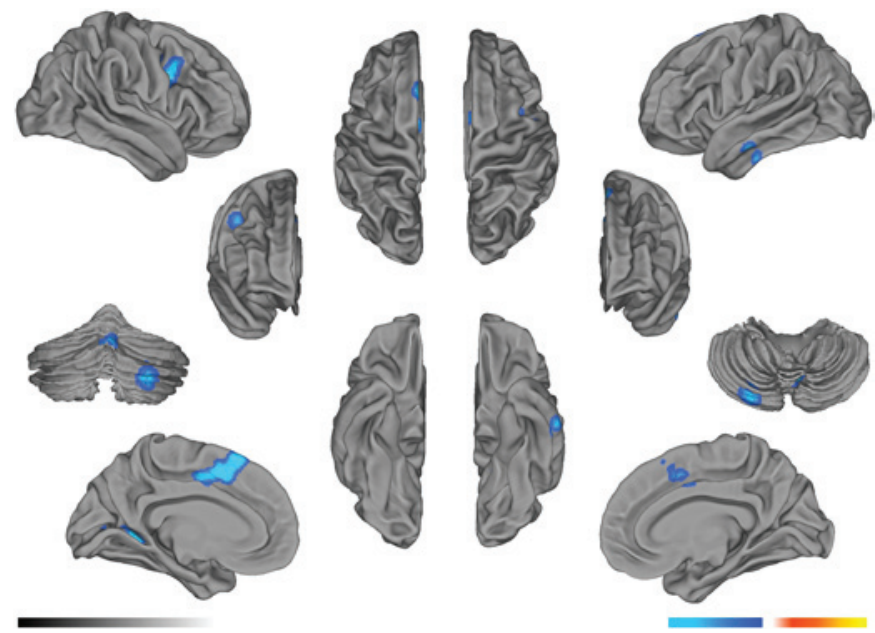

Figure 4. Compared with the pre-radiotherapy group, the gray matter volume significantly decreased in the bilateral frontal lobe, right occipital lobe, left parietal lobe, right temporal lobe and left cerebellum in the 0-6 month post-radiotherapy group $(\mathrm{P}<0.05)$.
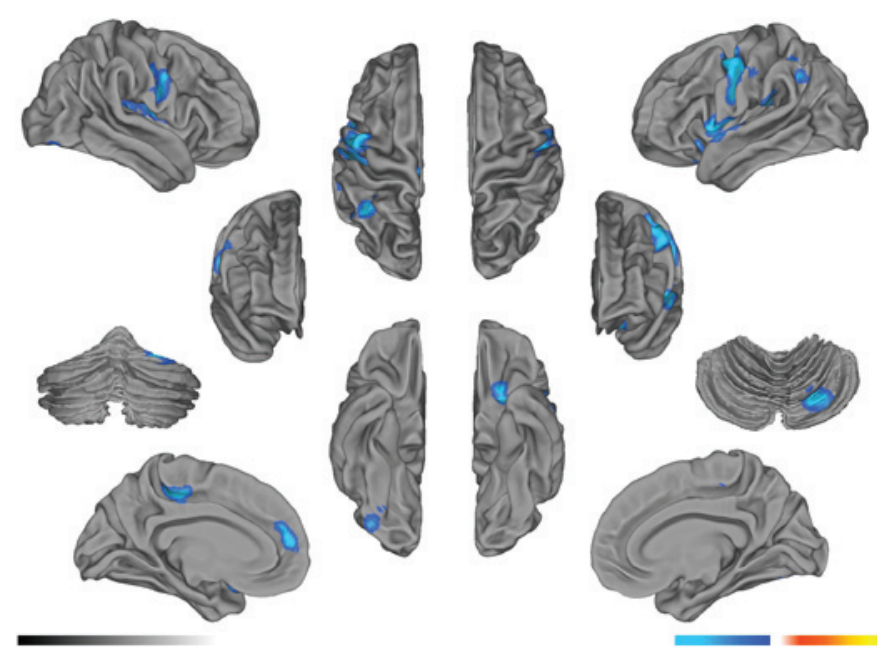

Figure 5. Compared with the pre-radiation group, the gray matter volume decreased significantly in the bilateral temporal lobe, parietal lobe, right frontal lobe and left cerebellum in the 6-12 month post-radiotherapy group $(\mathrm{P}<0.05)$.
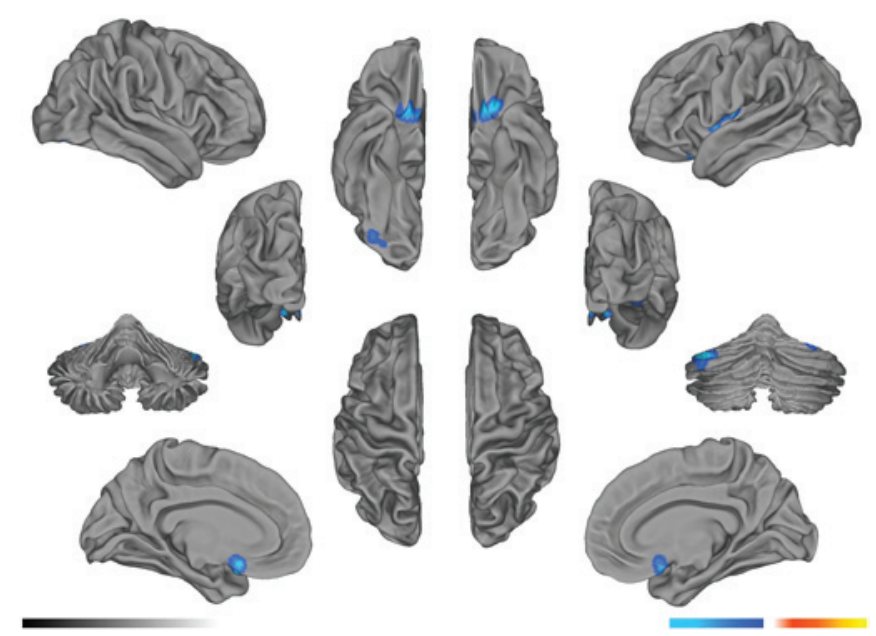

Figure 6. Compared with the pre-radiation group, the gray matter volume decreased significantly in the right temporal lobe, bilateral frontal lobe and bilateral cerebellum in the $>12$ month post-radiotherapy group $(\mathrm{P}<0.05)$. temporal lobe WM of patients remained markedly decreased after 12 months, indicating that early WM injury did not return to normal levels following RT and the temporal lobe may be the most seriously damaged area.

In the present study, the FA values markedly decreased in the parietal lobe, temporal lobe and cerebellar hemisphere which indicated that radiation directly or indirectly damaged the neural structure. There have been a number of hypotheses to explain this phenomenon including that radiation may damage the vascular endothelial cells and subsequently cause local brain tissue ischemia of the whole brain (20). Radiation may induce central nervous system autoimmune vasculitis, injuring the whole brain WM $(21,22)$. Thus, the microstructural alterations in the WM are dynamic and extensive in patients with NPC following RT.

Certain patients with normal conventional MRI scans exhibited markedly abnormal neurological symptoms, such as memory loss, following radiotherapy for nasalpharyngeal carcinoma in a previous study (23). In addition, a number of neural psychology test studies have revealed that patients with cranial irradiation experienced a decrease in cognitive function (24). Previous studies have been limited to the WM due to radiation sensitivity. However, serious radiation encephalopathy may involve the GM and glial cells, located in the GM, are sensitive to radiation. Additionally, the blood vessels of the GM are richer compared with that in the WM, which may be damaged by radiation; therefore, following RT, the GM of the brain may undergo alterations. Structural imaging using VBM is effective for evaluating GM volume alterations and has been widely used in the study of diseases where conventional imaging cannot be used, including drug addiction and depression $(25,26)$.

The GM volume alterations may be explained by a number of potential underlying pathophysiological mechanisms, including increases or decreases in neural or glial cells, cell size and exchanges between blood and tissue fluid (27). Although a limited number of neurons died following radiation, the dysfunction in axons and synapses may induce central nervous system damage (28). In addition, radiation may eliminate oligodendrocytes (29), resulting in a decrease in GM volume. The results of the present study identified that the brain GM volume decreased in the bilateral temporal lobe, bilateral cerebellar or bilateral frontal lobe and occipital lobe, which was consistent with a previous animal study, which demonstrated that irradiation caused early volume deficits in a number of brain regions in mice (30). The bilateral temporal lobe, bilateral cerebellum and bilateral occipital lobe may be included in the radiation field, which may be why the observed decreases in GM volumes were primarily induced by radiation. Additionally, a previous study demonstrated that chemotherapy may induce decreases in the frontal lobe GM volume in patients with breast cancer (31). Chemotherapy in combination with RT has previously be used to treat patients with NPC and therefore, the decrease in frontal lobe GM volume may result from chemotherapy. The results of the present study demonstrated that the alterations in the GM were widespread, complex and dynamic, and did not involve the temporal lobe only. The most drastic alterations and the most serious injury to brain tissue was observed between 0 and 6 months; however, after 1 year, patients recovered to a greater extent. 
The present study had a number of limitations. First, chemotherapy was not considered as a factor, as all the patients included in the present study received this. Secondly, the study lacked a healthy control group.

DTI and VBM were more sensitive compared with conventional MRI in determining radiation-induced brain damage and these two methods may be used in the long-term follow-up of patients with NPC following RT, to monitor alterations indicative of radiation-induced brain damage.

\section{References}

1. Shanmugaratnam K, Chan SH, de-Thé G, Goh JE, Khor TH, Simons MJ and Tye CY: Histopathology of nasopharyngeal carcinoma: Correlations with epidemiology, survival rates and other biological characteristics. Cancer 44: 1029-1044, 1979.

2. Sun Y, Zhou GQ, Qi ZY, Zhang L, Huang SM, Liu LZ, Li L, Lin AH and Ma J: Radiation-induced temporal lobe injury after intensity modulated radiotherapy in nasopharyngeal carcinoma patients: A dose-volume-outcome analysis. BMC Cancer 13: 397, 2013.

3. Lee AW, Law SC, Ng SH, Chan DK, Poon YF, Foo W, Tung SY, Cheung FK and Ho JH: Retrospective analysis of nasopharyngeal carcinoma treated during 1976-1985: Late complications following megavoltage irradiation. Br J Radiol 65: 918-928, 1992

4. Tang Y, Luo D, Rong X, Rong X, Shi X and Peng Y: Psychological disorders, cognitive dysfunction and quality of life in nasopharyngeal carcinoma patients with radiation-induced brain injury. PLoS One 7: e36529, 2012.

5. Glantz MJ, Burger PC, Friedman AH, Radtke RA, Massey EW and Schold SC Jr: Treatment of radiation-induced nervous system injury with heparin and warfarin. Neurology 44: 2020-2027, 1994.

6. Wang HZ, Qiu SJ, Lv XF, Wang YY, Liang Y, Xiong WF and Ouyang ZB: Diffusion tensor imaging and 1H-MRS study on radiation-induced brain injury after nasopharyngeal carcinoma radiotherapy. Clin Radiol 67: 340-345, 2012.

7. Xiong WF, Qiu SJ, Wang HZ and Lv XF: 1H-MR spectroscopy and diffusion tensor imaging of normal-appearing temporal white matter in patients with nasopharyngeal carcinoma after irradiation: Initial experience. J Magn Reson Imaging 37: 101-108, 2013.

8. Nelles M, Block W, Träber F, Wüllner U, Schild HH and Urbach H: Combined 3T diffusion tensor tractography and $1 \mathrm{H}-\mathrm{MR}$ spectroscopy in motor neuron disease. AJNR Am J Neuroradiol 29: 1708-1714, 2008.

9. Yokoyama K, Matsuki M, Shimano H, Sumioka S, Ikenaga T, Hanabusa K, Yasuda S, Inoue H, Watanabe T, Miyashita M, et al: Diffusion tensor imaging in chronic subdural hematoma: Correlation between clinical signs and fractional anisotropy in the pyramidal tract. AJNR Am J Neuroradiol 29: 1159-1163, 2008.

10. Zhuang L, Wen W, Zhu W, Trollor J, Kochan N, Crawford J, Reppermund S, Brodaty $\mathrm{H}$ and Sachdev P: White matter integrity in mild cognitive impairment: A tract-based spatial statistics study. Neuroimage 53: 16-25, 2010.

11. Chan YL, Leung SF, King AD, Choi PH and Metreweli C: Late radiation injury to the temporal lobes: Morphologic evaluation at MR imaging. Radiology 213: 800-807, 1999.

12. Norris AM, Carrington BM and Slevin NJ: Late radiation change in the CNS: MR imaging following gadolinium enhancement. Clin Radiol 52: 356-362, 1997.

13. Lv XF, Zheng XL, Zhang WD, Liu LZ, Zhang YM, Chen MY and Li L: Radiation-induced changes in normal-appearing gray matter in patients with nasopharyngeal carcinoma: A magnetic resonance imaging voxel-based morphometry study. Neuroradiology 56: 423-430, 2014.
14. Smith SM, Jenkinson M, Johansen-Berg H, Rueckert D, Nichols TE, Mackay CE, Watkins KE, Ciccarelli O, Cader MZ, Matthews PM and Behrens TE: Tract-based spatial statistics: Voxelwise analysis of multi-subject diffusion data. Neuroimage 31: 1487-1505, 2006.

15. Smith SM and Nichols TE: Threshold-free cluster enhancement: Addressing problems of smoothing, threshold dependence and localisation in cluster inference. Neuroimage 44: 83-98, 2009.

16. Valk PE and Dillon WP: Radiation injury of the brain. AJNR Am J Neuroradiol 12: 45-62, 1991.

17. Chong VF, Fan YF and Mukherji SK: Radiation-induced temporal lobe changes: CT and MR imaging characteristics. AJR Am J Roentgenol 175: 431-436, 2000.

18. Makki MI, Chugani DC, Janisse J and Chugani HT: Characteristics of abnormal diffusivity in normal-appearing white matter investigated with diffusion tensor MR imaging in tuberous sclerosis complex. AJNR Am J Neuroradiol 28: 1662-1667, 2007.

19. Kitahara S, Nakasu S, Murata K, Sho K and Ito R: Evaluation of treatment induced cerebral white matter injury by using diffusion-tensor MR imaging: Initial experience. AJNR Am J Neuroradiol 26: 2200-2206, 2005.

20. Russo C, Fischbein N, Grant E and Prados MD: Late radiation injury following hyperfractionated craniospinal radiotherapy for primitive neuroectodermal tumor. Int J Radiat Oncol Biol Phys 44: 85-90, 1999.

21. Xavier S, Piek E, Fujii M, Javelaud D, Mauviel A, Flanders KC, Samuni AM, Felici A, Reiss M, Yarkoni S, et al: Amelioration of radiation-induced fibrosis: Inhibition of transforming growth factor-beta signaling by halofuginone. J Biol Chem 279: 15167-15176, 2004.

22. Kim SH, Lim DJ, Chung YG, Cho TH, Lim SJ, Kim WJ and Suh JK: Expression of TNF-alpha and TGF-beta 1 in the rat brain after a single high-dose irradiation. J Korean Med Sci 17: 242-248, 2002

23. Parkin AJ and Hunkin NM: Memory loss following radiotherapy for nasal pharyngeal carcinoma-an unusual presentation of amnesia. Br J Clin Psychol 30: 349-357, 1991.

24. Cheung M, Chan AS, Law SC, Chan JH and Tse VK: Cognitive function of patients with nasopharyngeal carcinoma with and without temporal lobe radionecrosis. Arch Neurol 57: 1347-1352, 2000.

25. Özdemir Hİ, Eker MÇ, Zengin B, Yilmaz DA, İşman Haznedaroğlu D, Çınar C, Kitiş Ö, Akay A and Gönül AS: Gray matter changes in patients with deficit schizophrenia and non-deficit schizophrenia. Turk Psikiyatri Derq 23: 237-246, 2012.

26. Lai CH: Gray matter volume in major depressive disorder: A meta-analysis of voxel-based morphometry studies. Psychiatry Res 211: 37-46, 2013.

27. Schmidt-Wilcke T, Leinisch E, Straube A, Kämpfe N, Draganski B, Diener HC, Bogdahn U and May A: Gray matter decrease in patients with chronic tension type headache. Neurology 65: 1483-1486, 2005.

28. Shi L, Adams MM, Long A, Carter CC, Bennett C, Sonntag WE, Nicolle MM, Robbins M, D'Agostino R and Brunso-Bechtold JK: Spatial learning and memory deficits after whole-brain irradiation are associated with changes in NMDA receptor subunits in the hippocampus. Radiat Res 166: 892-899, 2006.

29. Tofilon PJ and Fike JR: The radioresponse of the central nervous system: A dynamic process. Radiat Res 153: 357-370, 2000.

30. Gazdzinski LM, Cormier K, Lu FG, Lerch JP, Wong CS and Nieman BJ: Radiation-induced alterations in mouse brain development characterized by magnetic resonance imaging. Int J Radiat Oncol Biol Phys 84: e631-e638, 2012.

31. McDonald BC, Conroy SK, Ahles TA, West JD and Saykin AJ: Gray matter reduction associated with systemic chemotherapy for breast cancer: A prospective MRI study. Breast Cancer Res Treat 123: 819-828, 2010. 